RA patients with MS. It was revealed that there are significant differences in the level of both ANGPTL3 ( $F=8.86, p=0.0034)$ and ANGPTL4 $(F=29.6, p<0.001)$ between RA patients with varying severity of metabolic disorders.

The study of the influence of several factors (MS and renal dysfunction) on the content of ANGPTL in RA patients showed that the presence of metabolic disorders had an insignificant effect on the parameters of ANGPTL3 in the groups of patients with optimal and slightly reduced GFR, acquiring significance only with a more intense decrease in GFR $(<59 \mathrm{ml} / \mathrm{min} / 1.73 \mathrm{~m} 2)$. These factors and their interactions explain the insignificant share of the variability in ANGPTL3 $\left(R^{2}=\right.$ 0.11 ), which indicates a low quality of the model.

There was a more pronounced difference in the level of ANGPTL4 in the presence of metabolic disorders in the groups of RA patients with varying degrees of renal dysfunction. The factors under consideration and their interactions do not make this model significant $(p=0.1)$, although they can explain a significant proportion of the variability in ANGPTL4 $\left(R^{2}=0.32\right)$.

We differentiated RA patients into patients with high or optimal GFR ( $\geq 89 \mathrm{ml}$ / $\mathrm{min}$ ) and patients with reduced GFR ( $<89 \mathrm{ml} / \mathrm{min}$ ) when combining groups of RA patients with varying degrees of renal dysfunction (group II and group III). Multivariate analysis of variance using the new characteristics showed a significant increase in ANGPTL 4 in the blood serum of RA patients with reduced GFR (F = $18.5, p<0.001)$ and severe metabolic changes $(F=24.2, p<0.001)$.

Thus, the concentration of ANGPTL4 in RA patients is directly influenced by two factors (renal dysfunction and the presence of MS), which can describe the variability of this sign in more than $30 \%$ of cases. The squared multiple correlation coefficient $\left(R^{2}\right)$ in this model is 0.33 .

Conclusion: ANGPTL type 4 should be considered as a key factor linking the development of the renal dysfunction in RA patients and the metabolic changes caused by rheumatoid inflammation.

Disclosure of Interests: None declared

DOI: 10.1136/annrheumdis-2021-eular.2657

\section{POS0547 METEOROLOGICAL VARIABLES HAVE DIFFERENT INFLUENCE ON CORE MEASURES OF DISEASE ACTIVITY IN PATIENTS WITH RHEUMATOID ARTHRITIS}

P. Mandl ${ }^{1}$, P. Studenic ${ }^{1}$, F. Alasti ${ }^{1}$, R. Kaltenberger ${ }^{2}$, A. Kerschbaumer ${ }^{1}$, T. Krennert ${ }^{3}$, J. S. Smolen ${ }^{1}$, D. Aletaha ${ }^{1} .{ }^{1}$ Medical University of Vienna, Rheumatology, Vienna, Austria; ${ }^{2}$ Central Institute for Meteorology and Geodynamics, Climate Research, Vienna, Austria; ${ }^{3}$ Central Institute for Meteorology and Geodynamics, Weather Forecast, Vienna, Austria

Background: The notion that weather conditions may influence the symptoms and course of rheumatic and musculoskeletal diseases goes back to ancient times. However, despite the seemingly obvious relationship, previous studies assessing how meteorological variables affect pain and disease activity have yielded contradictory results.

Objectives: To evaluate whether meteorological variables influence disease activity in patients with rheumatoid arthritis (RA).

Methods: We assessed correlations between clinical measures of disease activity (pain, patient global assessment [PGA], tender- and swollen 28 joint counts [TJC and SJC]) and individual meteorological variables: temperature (temp.), effective (eff.) temperature, saturation vapor pressure (SVP), absolute humidity $(A H)$, relative humidity $(\mathrm{RH})$, dew point, vapor pressure (VP) and precipitation. Assessments documented in the Care for Rheumatoid Arthritis database of our institution were matched with these meteorological variables on a daily basis for a period of 12 years between 2005 and 2017 and analyzed utilizing generalized estimating equations. Patients with $<5$ visits in the study period, those with $<1$ visit/quarter or with no pain $(\mathrm{VAS}=0$ ) in $\geq 3$ consecutive visits and those living outside of the catchment area were excluded. Patients were grouped into remission or low $(\leq 11)$ vs. moderate or high $(>11)$ disease activity based on the simplified disease activity index (SDAI).

Results: A total of 461 patients with an average disease duration at first visit of $5.7 \pm 7.4$ years, average age of $55.3 \pm 14.5$ years and a mean SDAI of $22.1 \pm 12.7$ were analyzed. Among patients with moderate or high disease activity, higher temp./eff. temp. and SVP were associated with lower pain, TJC and SJC (Figure 1); on the contrary, higher $\mathrm{RH}$ was associated with higher pain and higher SJC. In those in remission or low disease activity, higher $\mathrm{RH}, \mathrm{AH}$, VP or dew point were associated with lower PGA. Higher precipitation was associated with lower SJC.

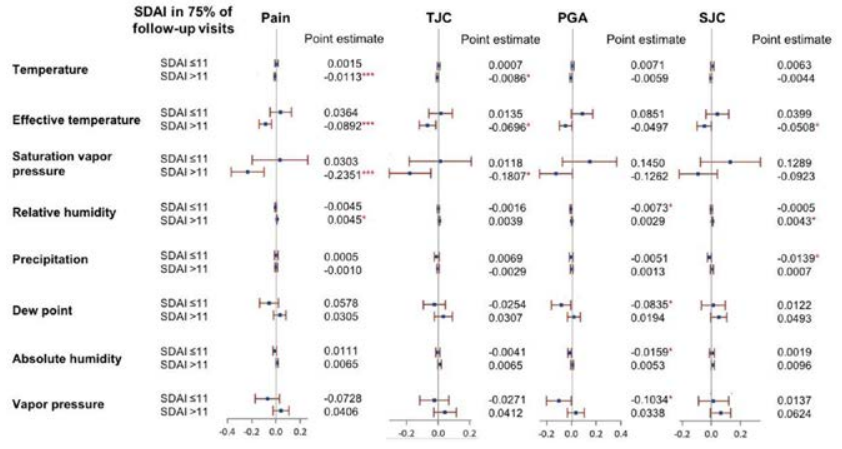

Figure 1. Association of meteorological parameters and clinical measures of disease activity. PGA: patient's global assessment, SDAl: simplified disease activity index, SJC: swollen joint count, TJC: tender joint count. ${ }^{*} p \leq 0.05,{ }^{\star \star}{ }^{*} p \leq 0.01,{ }^{\star \star \star} p \leq 0.001$

Conclusion: In this large association study of meteorological parameters with RA, both temperature and humidity parameters were modestly inversely associated with pain, TJC and SJC.

Disclosure of Interests: None declared

DOI: 10.1136/annrheumdis-2021-eular.2684

\section{\begin{tabular}{|l|l}
\hline POS0548 & OPTIMAL ASSESSMENT OF THE RHEUMATOID
\end{tabular} ARTHRITIS ANKLE AND HINDFOOT: A COMPARATIVE STUDY BETWEEN CLINICAL EXAMINATION AND ULTRASONOGRAPHY}

K. Maatallah ${ }^{1}, \underline{\text { H. Boussaa }}{ }^{1}$, H. Riahi ${ }^{2}$, H. Ferjani ${ }^{1}$, M. Habechi ${ }^{1}$, W. Triki ${ }^{1}$, D. Ben Nessib ${ }^{1}$, M. Bouaziz ${ }^{2}$, D. Kaffel ${ }^{1}$, W. Hamdi ${ }^{1}{ }^{1}$ Mohamed Kassab Institute of Orthopedics, Rheumatology, La Manouba, Tunisia; ${ }^{2}$ Mohamed Kassab Institute of Orthopedics, Radiology, La Manouba, Tunisia

Background: Foot disease is a common problem in rheumatoid arthritis (RA). Therapeutic decisions are often based on clinical examination (CE) alone, which can be adversely affected by factors such as deformity, obesity, and peripheral edema. Ultrasonography (US) has previously been shown to be more sensitive than CE for detecting synovitis and tenosynovitis in RA forefeet, but few data exist for the hindfoot and ankle.

Objectives: The aim of this study was to compare CE and US for the detection of hindfoot and ankle synovitis and tenosynovitis in patients with established RA. Methods: We conducted a cross-sectional study including patients with RA (ACR/EULAR 2010). Demographic data and disease parameters were collected. CE was performed by a rheumatologist for the presence or absence of tenderness, swelling, and mobility restriction of both ankles. The following tendons were examined for tenosynovitis: tibialis anterior (TA) and posterior (TP), fibularis longus (FL), and brevis (FB) (assessed together). In a second time, US examination of the tibiotalar, talonavicular, and subtalar joints and the same tendons as CE was performed by a blinded radiologist experienced in musculoskeletal imaging using a Philips HD11 device with a high-frequency linear transducer. The presence or absence of synovitis and tenosynovitis was recorded, and the composite synovitis score (power doppler / grayscale ultrasound (PDUS)) was measured for each joint. The US score of each patien was defined by the sum of the composite scores of the joints studied (0-30). A p-value $<0.05$ was considered significant.

Results: Sixty-two feet were examined in 31 RA patients (25 women and six men) with a mean age of $54.8 \pm 10.8$ years old [32-70]. The mean disease duration was $8.5 \pm 7.2$ years [1-37]. Rheumatoid Factor (RF) and Anti-Citrullinated Peptides Antibodies (ACPA) were positive in $61.3 \%$ and $83.8 \%$ of cases. The mean DAS28 ESR was 3.8 \pm 1.5 [0.6-7].

Clinical examination of ankles revealed tenderness in $57.4 \%$ of cases, swelling in $38.8 \%$ of cases, and restriction in the range of motion in $11.1 \%$ of cases. TA tenosynovitis was noted in $14.8 \%$ of cases, TP tenosynovitis in $22.2 \%$ of cases, and $\mathrm{FL}$ and $\mathrm{FB}$ tenosynovitis in $31.5 \%$ of cases.

US showed tibiotalar synovitis in $59.3 \%$ of cases, talonavicular synovitis in $64.8 \%$ of cases, and subtalar synovitis in $46.3 \%$ of cases. TA tenosynovitis was noted in $5.6 \%$ of cases, TP tenosynovitis in $22.2 \%$ of cases, and FB and FL tenosynovitis in $25 \%$ and $11.1 \%$ of cases respectively. 